\title{
Reification of galaxies: cognitive astrophysics and the multiwavelength inverse problem
}

\author{
Barry F. Madore \\ Carnegie Observatories, Pasadena, California, USA
}

Lessons learned in the history and philosophy of science have generally had little immediate impact on how we as individual astronomers conduct our research. And yet we do share many common views on how we undertake basic research, and how we translate observations and theory into communicable knowledge. In this introductory talk I will illustrate how we as extragalactic astronomers have already violated some of the basic tenets of what constitutes "science" as seen from a philosophical point of view, and I will predict what the future of astronomy as a science may soon look like. Simple examples of how we are already "cognitively closed" to many immediate and tangible aspects of the Universe will be given and some solutions to this dilemma will be proposed. We may be at a point in time where more data is not necessarily the best solution to our problems. Discovering that familiar concepts and even certain objects may not exist in the traditional sense of the word could provide a motivation for broadening our way of conceptualizing the extragalactic Universe, more as a continuum of processes and phase transitions rather than an assembly of discrete objects. Once again the Universe may be "forcing us to think". 